\title{
Prototyping: Major Advance in Surgical Planning and Customizing Prostheses in Patients with Bone Tumors of the Head and Neck
}

\author{
Terence P. Farias ${ }^{1}$, Fernando Luiz Dias ${ }^{1}$, Bruno Albuquerque Sousa ${ }^{1}$, Mário Sérgio Galvão², \\ Dênio Bispo ${ }^{1}$, Ana Carolina Pastl ${ }^{1}$ \\ ${ }^{1}$ Department of Head and Neck Surgery, Brazilian National Cancer Institute, Rio de Janeiro, Brazil; ${ }^{2}$ Department of Microsurgery \\ Reconstruction, Brazilian National Cancer Institute, Rio de Janeiro, Brazil. \\ Email: terencefarias@yahoo.com.br
}

Received April 25 $5^{\text {th }}, 2013$; revised May 30 ${ }^{\text {th }}, 2013$; accepted June $10^{\text {th }}, 2013$

Copyright (C) 2013 Terence P. Farias et al. This is an open access article distributed under the Creative Commons Attribution License, which permits unrestricted use, distribution, and reproduction in any medium, provided the original work is properly cited.

\begin{abstract}
Major resections of bone tumors of the head and neck, owing to their small dimensions and their proximity to the organs in this region, cause important functional and aesthetic problems that significantly compromise patients' quality of life. Therefore, therapeutic planning should always extend beyond the resection to include functional and aesthetic reconstruction, preferably immediately. Microsurgical reconstruction represents a significant advance, but has not yet been perfected. Rapid prototyping (RP) comes as a new technology for the purpose of assisting the surgeon in the visual and tactile aspects of surgery, providing diagnostic accuracy and increasing the success of surgical planning. The authors demonstrate the technological advances in the manufacture of customized mandibular prostheses with the assistance of RP and practical applications of these methods.
\end{abstract}

Keywords: Rapid Prototyping; Bone Tumors; Head Neck Tumors

\section{Introduction}

Major resections of bone tumors of the head and neck, owing to their small dimensions and their proximity to the organs in this region, cause important functional and aesthetic problems that significantly compromise patients' quality of life. Therefore, therapeutic planning should always extend beyond the resection to include functional and aesthetic reconstruction, preferably immediately [1,2]. Microsurgical reconstruction represents a significant advance, but has not yet been perfected. Rapid prototyping (RP) comes as a new technology for the purpose of assisting the surgeon in the visual and tactile aspects of surgery, providing diagnostic accuracy and increasing the success of surgical planning, and thus, improving outcomes and reducing complications, risks, operative time and the overall cost of treatment [3-5].

$\mathrm{RP}$ is a constructive, additive process used to obtain physical prototypes from a digital three-dimensional model. Based on this three-dimensional model, RP systems build prototypes by successively adding fine layers of specific materials [6,7]. The introduction of RP tech- nology in medicine is relatively recent. With progress in technological processes to acquire medical images, including computerized tomography (CT) and magnetic resonance imaging (MRI), high quality, three dimensional images that permit the visualization, manipulation and analysis of anatomical structures are possible to generate. These technological advances, used in conjunction with new computerized analysis techniques, yield clinically relevant information from the original image [810].

Another applicability of RP is in the manufacture of customized prostheses being widely used in cranioplasty (Figure 1). In this context, Dean et al conducted a study to replace current manual methods for preoperative production of large-format $\left(>100 \mathrm{~cm}^{2}\right)$ cranioplasties with a system for computer-aided design and direct computer-aided manufacture of the implant's shape. The authors manually generated cranial implants produced for five patients and compared with implants resulting from computer-based method. Therefore, the study concluded that computer-generated implants were better fitting and more cosmetically suitable than the manually generated 

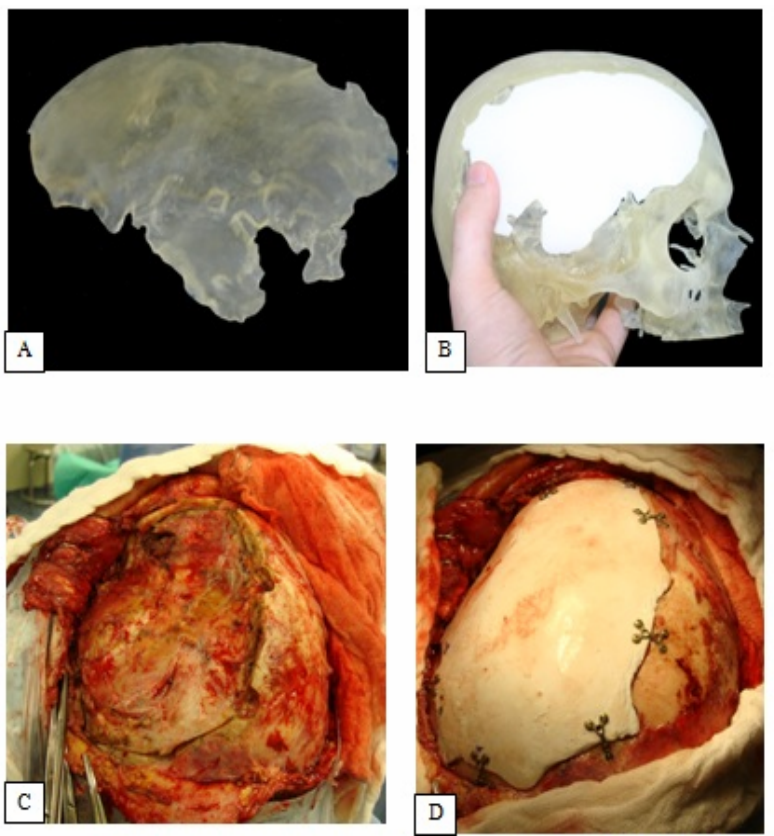

Figure 1. Symmetric reconstruction of the calvaria after tumor removal using rapid prototyping models.

skull plates received by these patients and a well-fitting implants are more likely to protect the brain from trauma and infection [11].

More recently Haturu et al. conducted a study to assess quantitatively whether a symmetric reconstruction of the calvaria could be achieved using 3-dimensional (3D) custom-made implants and to examine any complications caused by the cranioplasty. The study concluded that the custom-made implants for cranioplasty showed a significant improvement in morphology and the implants may be very useful for repairing large and complexshaped cranial defects [12]. A similar study was performed by Chrzan et al. with the aim to assess the clinical usefulness of RP in cranioplasties prosthesis manufacturing. The authors emphasize the importance of RP in reduce the time needed for cranioplasty neurosurgery and improve the prosthesis fitting. Such technologies may utilize data obtained by commonly used spiral CT scanners and the manufacturing of individually adjusted prostheses should be commonly used in patients planned for cranioplasty with synthetic material [13]. Gerber et al. use RP in cranioplasties and believe that this technique results in excellent cosmetic outcome with reduced surgery time and reduced manufacturing costs [14].

Other promising situation is located in the construction of customized prosthesis for reconstruction of the temporomandibular joint and condyle in degenerative disorders. Deshmukh et al. performed a study using RP to fabricate a successful implant for temporomandibular joint disorder patients who could not be treated through conventional surgeries. The authors obtained a successful treatment to the deformed mandible and the mandible joints. The RP allows to customise and to accurately fabricatie the implant. Advantages of this approach are that the physical model of the implant was tested for stability before the implantation, the surgeon can plan and rehearse the surgery in advance, it is a less invasive and less time-consuming surgical procedure [15].

In this context, Keller et al. conducted a prospective study in patients with osteoarthritis of the temporomandibular joint and concluded that temporomandibular joint hemiarthroplasty with custom metal fossa/eminence prosthesis provides satisfactory clinical and functional outcomes when used for advanced osteoarthritis in patients with focal joint pain secondary to computed tomographically documented joint pathology [16].

Technological advances in the manufacture of customized mandibular prostheses with the assistance of RP have been popularized throughout the world and many specialized centers have already adopted this technique as the gold standard. Singare et al. developed a study with these purpose and perform a computer-assisted prefabricated implant design and manufacturing system to improve the aesthetic outcome in chin surgery. In practical implications, this method not only demonstrates the significant progress in the reconstruction of chin defects using RP, compared with the conventional methods of chin augmentation surgery, but also provides natural geometrical prosthesis contour design and accurate fabrication and precise fitting of the prosthesis. The advantages of using this technique are that the physical model of the implant is fitted on the skull model so that the surgeon can plan and rehearse the surgery in advance and a less invasive surgical procedure and less time-consuming reconstructive and an adequate aesthetic can result [17]. In addition, this method improves the reconstructive surgery and reduces the risk of a second intervention decreasing psychological stress of the patient [18].

Another application is the use of RP for functional mandibular reconstruction. Zhou et al. used a custommade titanium bone-grafting tray packed with autologous iliac bone evaluated esthetically and functionally a mandibular discontinuity defect. The authors concluded that the use of a customized technique using reverse engineering, computer-aided design, and rapid prototyping tray containing autologous cancellous bone is a potentially powerful grafting technique for the reconstruction of mandibular discontinuity defects [19].

Our group recently used the RP to custom fabricate an almost total mandibular prosthesis for reconstruction of bone defects in salvage surgery for advanced oral cancer obtaining excellent functional and aesthetic results. (Figure 2). 

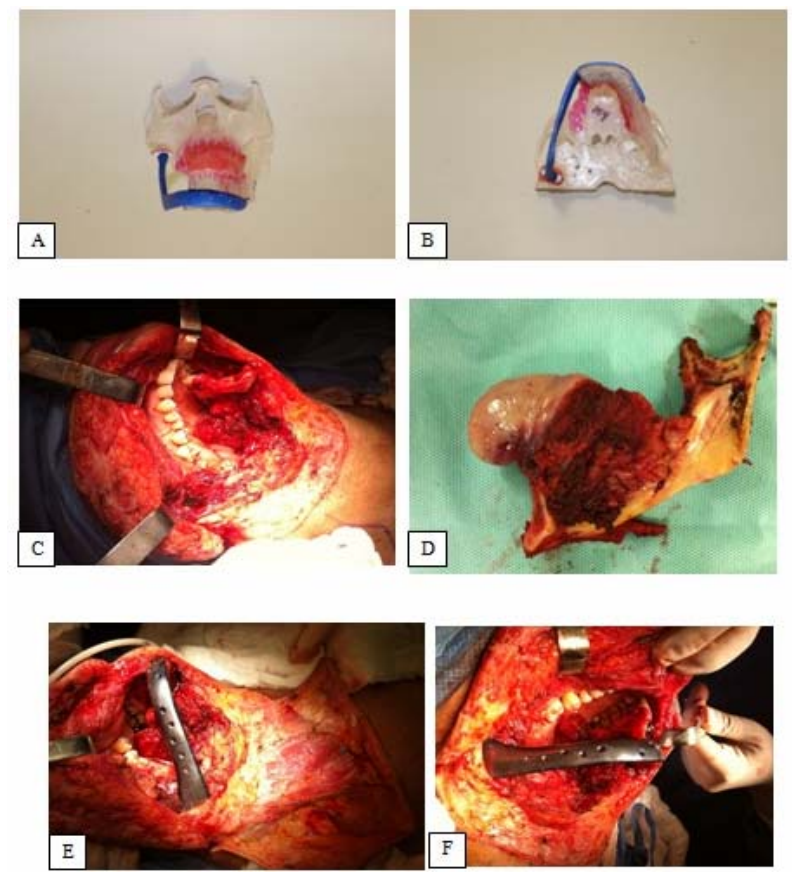

Figure 2. Reconstruction of mandibular defects in salvage surgery for advanced oral cancer using almost total titanium plate prototyped.

\section{Material and Methods}

In the Head and Neck Department of the Brazilian National Cancer Institute patients with a primary diagnosis of a mandibular tumor or with secondary involvement; which had not received any previous oncologic treatment and had no contraindications for surgical treatment were selected to tumor resection and microsurgical reconstruction with the use of prototyping.

All of the patients, independent of gender, underwent segmental mandibulectomy with more than $3 \mathrm{~cm}$ of bone loss. Therefore, a microsurgical reconstruction was necessary. In addition to preoperative exams, the patients underwent tomography of the primary tumor site and donor bone reconstruction (iliac crest and fibula).

The tomographic images of the prototype group were recorded on a $\mathrm{CD}$ and sent to the prototyping machine. The acquired images were studied at various angles and used to determine whether the iliac crest or the fibula would be the best option for reconstruction. At this time, using the three-dimensional images on the computer, the surgeons were able to rotate study and choose the best donor area (iliac crest or fibula) and draw on the bone selected for prototyping.

The prototypes were composed of gypsum, cyanoacrylate, and ZP150 and processed using prototyping machines $3 \mathrm{D} 30$ and Z510, with the binder ZB60, to perform the process of hardening and agglutination.

Regardless of previously reported procedures for per- forming the design prototyping, all of the patients underwent the "gold-standard" treatment that is routinely applied to patients with similar diagnoses and staging: surgical resection of the primary tumor with ample margins together with micro-surgical reconstruction. There was no change in the type of anesthesia commonly used in these cases. General anesthesia was used, with the standard anesthetic technique. The prototype underwent an operation one day before the surgery, and the material was sterilized 24 hours in advance (Figures 3 and $\mathbf{4}$ ).

The tumor resection and bone flap were made simultaneously by the head and neck and plastic surgeons, respectively, once the plastic surgery team already knew the defect to be reconstructed, the bone flap was harvested exactly fit the defect, leading to no waste and reducing the operative time.

The aesthetic outcome of the patients was evaluated by 3 surgeons and 3 non-medical professionals, who examined postoperative photos of patients prototyped and no-prototyped. The photos were given a grade of 0 to 10 with respect to mandibular symmetry, bulges, depresssions, and facial harmony. The grades reflected subjecttive evaluations [very good $(9-10)$, good $(7-8)$, regular $(5-6)$, bad $(3-4)$, and unacceptable $(1-2)]$. The evaluators were blinded to the prototyping status of the patients. All patients were photographed in the second month after surgery, when they were healed with no edema and before the start of radiotherapy. Only one
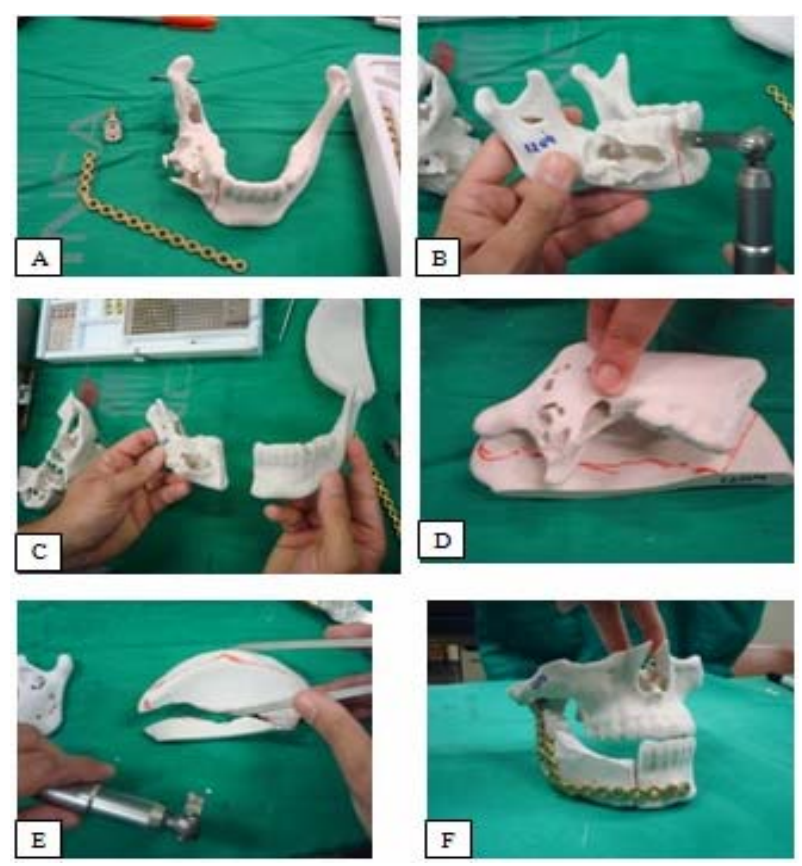

Figure 3. Prototype from the donor bone to be used for reconstructing the resected tumor biomodel. The size of the flap to be taken from the iliac crest could be determined accurately. 


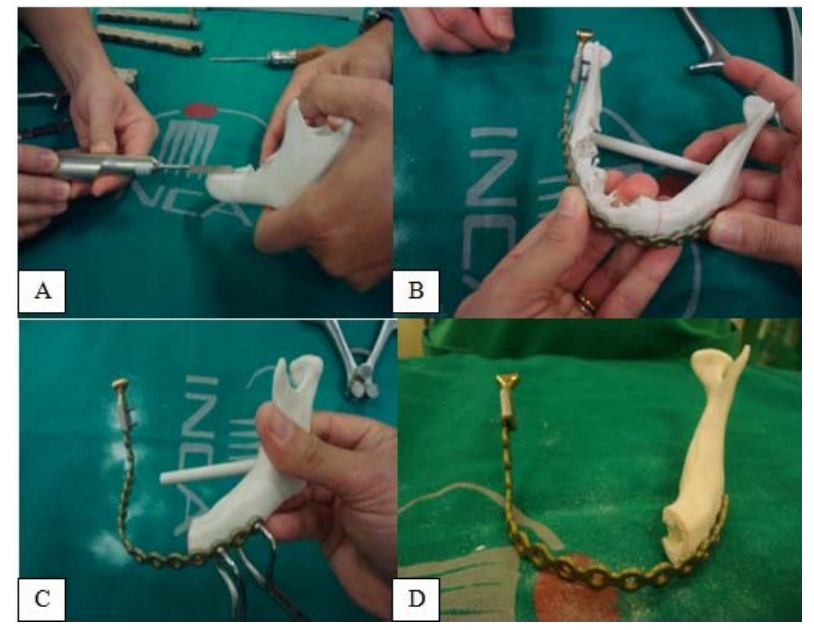

Figure 4. The surgery images from the molding prototype of the mandible with the tumor and the final appearance after placing the plates and screws. Observe the design of the prototype with a rod between the inner angles of the mandible stabilizing the occlusion after tumor resection.

patient was photographed with a slight orocutaneous fistula that did not result in distortion of facial symmetry. The opinions of the evaluators were analyzed by the Kappa index (with value of agreement -0.64) to statisticcally measure the inter-rater agreement for qualitative items between the groups.

\section{Results}

As results, 17 patients were operated with assist of prototyping. In patients for whom prototyping was used $18.18 \%$ were female, and $81.82 \%$ were male. The patients' ages ranged from 9 to 74 years with an average of 39.57 years. The most frequent histological tumor type was squamous cell carcinoma, followed by ameloblastoma. The other tumor types included the following: fibroxanthoma, keratocystic odontogenic tumor, mucoepidermoid carcinoma, sarcoma, embryonic rhabdomyosarcoma, adenoid cystic carcinoma and giant cell reparative granuloma.

The biomodels were used for mock operations one day before the patients' actual surgery. The mean duration of surgery using the biomodels was 115 minutes, ranging from 40 to 155 minutes. At this time, the plates were molded according to the defect induced in the biomodel, and the bone flap was made, fractured and adapted according to best practices. The appropriate screws for the bone thickness were selected (Figure 5). The prototypes were important for selecting the appropriate screws. A better anatomical understanding of the tumor was obtained using the prototype, which significantly aided in the patient's surgery. In addition, we were able to measure the size of the necessary bone flap with the aim to
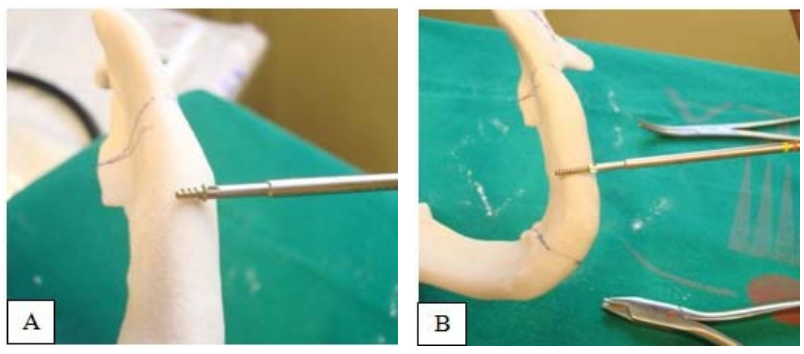

Figure 5. The selection of screws to be used for plate fixation (note the incorrect choice of screw in image $A$, which was corrected in image $B$ ).

decrease excess bone loss.

Upon completion of the biomodel surgery, the plates were molded, and the screws and saws were selected. These items were sterilized for use in the patients' surgeries the following day. The biomodel is not sterilizable, being protected with a sterile adhesive and used as an intraoperative mold to be harvested on the day of surgery.

The use of a rod between the inner angles of the mandible in the biomodel facilitated plate molding. This addition was very important because the biomodel did not lose the occlusion but rather maintained the occlusion for a longer period of time. The rod was used only in the biomodel. In surgery, we used intermaxillary blocking screws.

The choice of the bone flap was made via computer software that simulates the surgery by comparing reconstruction with the iliac crest or fibula. In cases of tumors affecting the central arch, we used the fibula with two osteotomies. In tumors that affected the body and ramus, we used the iliac crest.

The average aesthetic grade for the patients in the prototype group was 7.7 and 9.2 (medical and non-medical, respectively) versus 6 and 8.8 (medical and nonmedical, respectively) in patients without prototyping. The concordance of opinion among the reviewers was validated using the Kappa index $(-0.64)$, which clearly indicated that the best aesthetic results were obtained in patients for whom the prototyping aid was used prior to surgery (Figure 6).

\section{Discussion}

RP is becoming an essential technology to speed the development of new products. The use of prototyping allows for errors to be detected in the initial phases of the project. This feature enables the launch of a new product and increases the product's competitiveness [20]. The use of RP technologies in medicine today is a reality, and RP can be used in various types of surgical interventions. The investigations and ongoing developments represent 

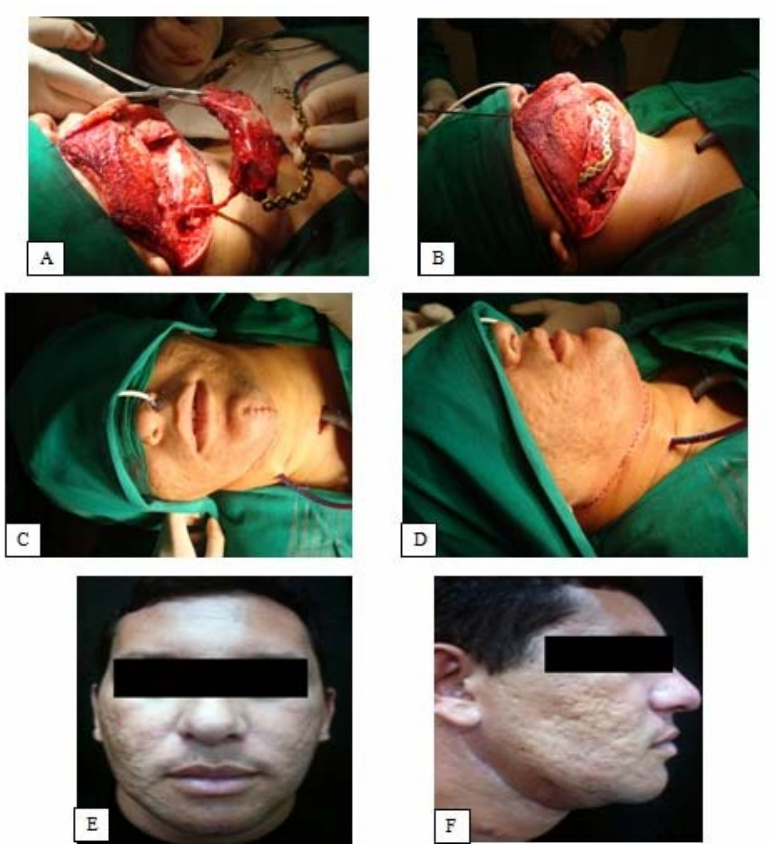

Figure 6. Perioperative in patient of the prototyped group with exceptional aesthetic and functional outcomes after surgery.

exciting innovations in the field of surgery [21].

The first applications of prototyping in maxillo-facial surgery were documented by Brix et al. in 1985 and Mankovich et al. in 1990. In 1994, Anderl et al. reported the use of a stereolithographic model in corrective surgery for a great cleft palate in a child at the University of Innsbruck [22]. In 1995, Bill et al. reported the use of prototyping to facilitate the reconstruction of a complex cranial defect in a patient with sequelae of infection after excision of a meningioma [23].

Some advantages for the use of RP in the medical field include the following: surgical simulation; making surgical guides on the bone; reducing surgical risk; reducing time and hospital costs; making personalized prostheses; using the opposite side to establish symmetry; planning with the patient at a distance; and aiding in documentation [24].

In our department we found that with the prototype, a better understanding of the tumor anatomy was possible. The time for tumor resection, by the same surgeon, knowing exactly where to perform the osteotomies, was relatively lower. Bisdas and Essig are also in agreement with our conclusion that the use of biomodels allow surgeons to "see and touch" to better understand and more accurately control procedures, such as cutting and perforations, during surgery $[25,26]$.

The comfort that prototyping affords the surgeon is undeniable, given that the molds have already been created, the screws have been preselected, and the cuts to be made in the mandible, iliac crest or fibula have already been defined by working with the prototype the day before.

The aesthetic outcome in the prototype group was better than the non-prototype group. This favorable outcome represents the result of an accurate pre-operative study with the aid of a computer, surgery on the previous biomodel, plate adaptation without surgical stress, and the team assessment 1 day prior to surgery, including studying of the case and operating on the biomodel.

Although the model construction is expensive, its use in pre-operative planning substantially reducing the operating time. Reducing the operating time is important given that the costs of the operating room represent 30 to $40 \%$ of the average surgical expenses [27].

RP has been used by several centers. One of the major statistics was reported by Hanasono et al. in a very well conducted study that assessed the use of computer-assisted design and RP modeling to improve the speed and accuracy of mandibular reconstruction in 38 subjects who underwent fibula free flap mandibular reconstructtion. The study showed that the prototype group had a reduced operative time and a better anatomical and functional accommodation bone flap and concluded that the computer-assisted design and RP modeling have the potential to increase the speed and accuracy of mandibular reconstruction. The authors also believe that these technologies are particularly useful for cases in which the original architecture of the mandible has been distorted or destroyed [28].

The method is being increasingly used, even in children as reported by Edwards for use in reconstructive surgery, bone flap shaping, distraction osteogenesis, orthognathic surgery and craniomaxillofacial surgery [29]. A great proposal by Bhumiratana for the future is to use $\mathrm{RP}$ in conjunction with tissue engineering, where the engineering of bone grafts customized to the patient and the specific clinical condition would revolutionize the way we currently treat craniofacial defects [30].

Levine et al. discussed RP with great enthusiasm, describing the technology as state of the art. The authors illustrate the ease by which virtual surgery, computeraided design and manufacturing can be used by the craniomaxillofacial surgeon to create tremendously accurate postoperative results and provide confidence with even the most complex three-dimensional bony reconstructions. The study reviews the application of these techniques in mandibular reconstruction, orthognathic surgery, maxillofacial trauma, and temporomandibular joint reconstruction [31].

In conclusion, the use of prototyping tended to reduce the surgical time because the premolded plates and previous selection of screws facilitated the surgery, reducing 
the operating time. There was a decrease in the size of the bone flap taken for reconstruction, decreasing morbidity at the donor area of the bone flap and was observed better aesthetic results in the patients who underwent operations that used prototyping.

\section{Acknowledgements}

We would like to thank the Renato Archer Research Center, Campinas, São Paulo, Brazil for contributing to the manufacturing of the prototypes.

\section{REFERENCES}

[1] D. Yousem, K. Gad and R. Tufano, "Resectability Issues with Head and Neck Cancer," AJNR, Vol. 27, No. 10, 2006, pp. 2024-2036.

[2] J. Boyd, "Use of Reconstruction Plates in Conjuction with Soft-Tissue Free Flaps for Oromandibular Reconstruction," Clinics in Plastic Surgery, Vol. 21, No. 1, 1994, pp. 69-77.

[3] J. Winder and R. Bibb, "Medical Rapid Prototyping Technologies: State of the Art and Current Limitations for Application in Oral and Maxillofacial Surgery," Journal of Oral and Maxillofacial Surgery, Vol. 63, No. 7, 2005, pp. 1006-1015. doi:10.1016/j.joms.2005.03.016

[4] R. Bibb, Z. Taha, R. Brown and D. Whight, "Development of a Rapid Prototyping Design Advice System," Journal of Intelligent Manufacturing Vol. 10, No. 3, 1999, pp. 331-339. doi:10.1023/A:1008920512663

[5] J.Choi, H. Choi, N. Kim, et al., "Analysis of Errors in Medical Rapid Prototyping Models," International Journal of Oral and Maxillofacial Surgery, Vol. 31, No. 1, 2002, pp. 23-32. doi:10.1054/ijom.2000.0135

[6] W. Yeong, C. Chua and K. Leong, "Rapid Prototyping in Tissue Engineering: Challenges and Potencial," Trends in Biotechnology, Vol. 22, No. 12, 2004, pp. 643-652. doi:10.1016/j.tibtech.2004.10.004

[7] D. Ma, F. Lin and C. Chua, "Rapid Prototyping Applications in Medicine. Part 1: NURBS-Based Volume Modelling," International Journal of Advanced Manufacturing Technology, Vol. 18, No. 2, 2001, pp. 103-117. doi:10.1007/s001700170081

[8] C. Kai, C. Meng, L. Ching, et al., "Rapid Prototyping Assisted Surgery Planning," International Journal of Advanced Manufacturing Technology, Vol. 14, No. 9, 1998, pp. 624-630. doi:10.1007/BF01192281

[9] C. Klug, K. Schicho, O. Ploder, et al., "Point-to-Point Computer-Assisted Navigation for Precise Transfer of Planned Zygoma Osteotomies from the Stereolithographic Model into Reality," Journal of Oral and Maxillofacial Surgery, Vol. 64, No. 3, 2006, pp. 550-559. doi:10.1007/BF01192281

[10] J. Martin and F. Keim, "Using Prototyping in Planning Microsurgical Reconstruction of the Mandible," Journal of Cranio-Maxillofacial Surgery, Vol. 14, No. 4, 2011, pp. 225-228.
[11] D. Dean, K. Min and A. Bond, "Computer Aided Design of Large-Format Prefabricated Cranial Plates," Journal of Craniofacial Surgery, Vol. 14, No. 6, 2003, pp. 819-832. doi:10.1097/00001665-200311000-00002

[12] H. Rotaru, H. Stan, I. Florian, et al., "Cranioplasty with Custom-Made Implants: Analyzing the Cases of $10 \mathrm{~Pa}-$ tients," Journal of Oral and Maxillofacial Surgery, Vol. 70, No. 2, 2012, pp. 169-176. doi:10.1016/j.joms.2011.09.036

[13] R. Chrzan, A. Urbanik, K. Karbowski, M. Moskala, J. Polak and M. Pyrich, "Cranioplasty Prosthesis Manufacturing Based on Reverse Engineering Technology," Medical Science Monitor, Vol. 18, No. 1, 2012, pp. 1-6. doi:10.12659/MSM.882186

[14] N. Gerber, L. Stieglitz, M. Peterhans, et al., "Using Rapid Prototyping Molds to Create Patient Specific Polymethylmethacrylate Implants in Cranioplasty," Engineering in Medicine and Biology Society (EMBC), 2010 Annual International Conference of the IEEE, Buenos Aires, 31 August-4 September 2010, pp. 3357-3360.

[15] T. Deshmukh, A. Kuthe, S. Chaware, V. Bagaria and D. Ingole, "A Novel Rapid Prototyping and Finite Element Method-Based Development of the Patient-Specific Temporomandibular Joint Implant," Computer Methods in Biomechanics and Biomedical Engineering, Vol. 15, No. 4, 2012, pp. 363-370. doi:10.1080/10255842.2010.538385

[16] E. Keller, E. Baltali, X. Liang, K. Zhao, M. Huebner and K. An, "Temporomandibular Custom Hemijoint Replacement Prosthesis: Prospective Clinical and Kinematic Study," Journal of Oral and Maxillofacial Surgery, Vol. 70, No. 2, 2012, pp. 276-288. doi:10.1016/j.joms.2011.06.202

[17] S. Singare, L. Dichen, L. Bingheng, G. Zhenyu and L. Yaxiong, "Customized Design and Manufacturing of Chin Implant Based on Rapid Prototyping," Rapid Prototyping Journal, Vol. 11, No. 2, 2005, pp. 113-118. doi:10.1108/13552540510589485

[18] S. Singare, L. Yaxiong, L. Dichen, L. Bingheng, H. Sanhu and L. Gang, "Fabrication of Customised MaxilloFacial Prosthesis Using Computer-Aided Design and Rapid Prototyping Techniques," Rapid Prototyping Journal, Vol. 12, No. 4, 2006, pp. 206-213. doi:10.1108/13552540610682714

[19] L. Zhou, J. Zhao, H. Shang, et al., "Reconstruction of Mandibular Defects Using a Custom-Made Titanium Tray in Combination with Autologous Cancellous Bone," Journal of Oral and Maxillofacial Surgery, Vol. 69, No. 5, 2011, pp. 1508-1518. doi:10.1016/j.joms.2010.06.185

[20] Y. Lin, S. Zhang, X. Chen and C. Wang, "A Novel Method in the Design and Fabrication of Dental Splints Based on 3D simulation and Rapid Prototyping Technology," International Journal of Advanced Manufacturing Technology, Vol. 28, No. 10, 2006, pp. 919-922. doi:10.1007/s00170-004-2197-1

[21] L. Cunningham, M. Madsen and G. Peterson, "Stereolithographic Modeling Technology Applied to Tumor Resection," Journal of Oral and Maxillofacial Surgery, 
Vol. 63, No. 6, 2005, pp. 873-878. doi:10.1016/j.joms.2005.02.027

[22] H. Anderl, D. Nedden, K. Twerdy, et al., "CT-Guided Stereolithography as a New Tool in Craniofacial Surgery," British Journal of Plastic Surgery, Vol. 47, No. 1, 1994, pp. 60-64. doi:10.1016/0007-1226(94)90121-X

[23] J. Bill, J. Reuther, W. Dittmann, et al., "Stereolithographyin Oral and Maxillofacial Operation Planning," International Journal of Oral and Maxillofacial Surgery, Vol. 24 , No. 1,1995 , pp. 98-103. doi:10.1016/S0901-5027(05)80869-0

[24] L. Zhao, P. Patel and M. Cohen, "Application of Virtual Surgical Planning with Computer Assisted Design in Maxillofacial Surgery," Archives of Plastic Surgery, Vol. 39, No. 4, 2012, pp. 309-316. doi:10.5999/aps.2012.39.4.309

[25] T. Bisdas and O. Teebken, "Future Perspectives for the Hole of 3-D Rapid Prototyping Aortic Biomodels in Vascular Medicine," VASA, Vol. 40, No. 6, 2011, pp. 427428. doi:10.5999/aps.2012.39.4.309

[26] H. Essig, M. Rana, H. Kokemueller, et al., "Pre-Operative Planning for Mandibular Reconstruction-A Full Digital
Planning Workflow Resulting in a Patient Specific Reconstruction," Head \& Neck Oncology, Vol. 3, No. 45, 2011, p. 45. doi:10.5999/aps.2012.39.4.309

[27] A. Macario, "What Does One Minute of Operating Room Time Cost?" Journal of Clinical Anesthesia, Vol. 22, No. 4, 2010, pp. 233-236. doi:10.5999/aps.2012.39.4.309

[28] M. Hanasono and R. Skoracki, "Computer-Assisted Design and Rapid Prototype Modeling in Microvascular Mandible Reconstruction," Laryngoscope, Vol. 123, No. 3, 2013, pp. 597-604. doi:10.5999/aps.2012.39.4.309

[29] S. Edwards, "Computer-Assisted Craniomaxillofacial Surgery," Oral \& Maxillofacial Surgery Clinics of North America, Vol. 22, No. 1, 2010, pp. 117-134. doi:10.1016/j.coms.2009.11.005

[30] S. Bhumiratana and G. Novakovic, "Personalized Human Bone Grafts for Reconstructiong Head and Face," Stem Cells Translational Medicine, Vol. 1, No. 1, 2012, pp. 6469.

[31] J. Levine, A. Patel, P. Saadeh and D. Hirsch, "Computer-Aided Design and Manufacturing in Craniomaxillofacial Surgery: The State of the Art," Journal of Craniofacial Surgery, Vol. 23, No. 1, 2012, pp. 288-293. doi:10.1097/SCS.0b013e318241ba92 